\title{
Relationship between Abdominal Pressure, Pulmonary Compliance, and Cardiac Preload in a Porcine Model
}

\author{
Joost Wauters, ${ }^{1}$ Piet Claus, ${ }^{2}$ Nathalie Brosens, ${ }^{1}$ Myles McLaughlin, ${ }^{2}$ Greet Hermans, ${ }^{1}$ \\ Manu Malbrain, ${ }^{3}$ and Alexander Wilmer ${ }^{1}$ \\ ${ }^{1}$ Medical Intensive Care Unit, University Hospital Gasthuisberg, Herestraat 49, 3000 Leuven, Belgium \\ ${ }^{2}$ Cardiac Imaging, University Hospital Gasthuisberg, 3000 Leuven, Belgium \\ ${ }^{3}$ Intensive Care Unit, Ziekenhuis Netwerk Antwerpen, Campus Stuivenberg, 2060 Antwerpen, Belgium
}

Correspondence should be addressed to Joost Wauters, joost.wauters@med.kuleuven.be

Received 29 August 2011; Accepted 31 October 2011

Academic Editor: Bart L. De Keulenaer

Copyright ( $\odot 2012$ Joost Wauters et al. This is an open access article distributed under the Creative Commons Attribution License, which permits unrestricted use, distribution, and reproduction in any medium, provided the original work is properly cited.

\begin{abstract}
Rationale. Elevated intra-abdominal pressure (IAP) may compromise respiratory and cardiovascular function by abdominothoracic pressure transmission. We aimed (1) to study the effects of elevated IAP on pleural pressure, (2) to understand the implications for lung and chest wall compliances and (3) to determine whether volumetric filling parameters may be more accurate than classical pressure-based filling pressures for preload assessment in the setting of elevated IAP. Methods. In eleven pigs, IAP was increased stepwise from 6 to $30 \mathrm{mmHg}$. Hemodynamic, esophageal, and pulmonary pressures were recorded. Results. 17\% (end-expiratory) to $62 \%$ (end-inspiratory) of elevated IAP was transmitted to the thoracic compartment. Respiratory system compliance decreased significantly with elevated IAP and chest wall compliance decreased. Central venous and pulmonary wedge pressure increased with increasing IAP and correlated inversely $(r=-0.31)$ with stroke index (SI). Global end-diastolic volume index was unaffected by IAP and correlated best with SI $(r=0.52)$. Conclusions. Increased IAP is transferred to the thoracic compartment and results in a decreased respiratory system compliance due to decreased chest wall compliance. Volumetric filling parameters and transmural filling pressures are clearly superior to classical cardiac filling pressures in the assessment of cardiac preload during elevated IAP.
\end{abstract}

\section{Introduction}

Elevated intra-abdominal pressure (IAP) is commonly encountered in critically ill patients as a result of abdominal diseases or conditions associated with bowel distention, ascites, peritonitis, hemoperitoneum, or trauma [1]. Based on recent consensus definitions, intra-abdominal hypertension (IAH) is diagnosed as a consistently increased IAP value of at least $12 \mathrm{mmHg}[2,3]$. Previous studies demonstrated negative effects of elevated IAP on respiratory function: a reduction in respiratory system compliance and increased airway pressures through elevation of the diaphragm [4-7]. In order to differentiate between the contribution of lung and chest wall in decreased respiratory system compliance, pleural pressure must be measured. The first aim of the present study was to analyze the effects of elevated IAP on pleural pressures at different stages of the respiratory cycle and the implications for measurements of lung or chest wall compliance and abdomino-thoracic pressure transmission.

Cardiovascular consequences of IAH include a reduction in cardiac output and stroke volume, resulting from both a decreased venous return and an increase in systemic vascular resistance $[4,8]$. Since central venous pressure (CVP) and pulmonary artery occlusion pressure (PAOP) typically increase with rising IAP in combination with decreasing stroke volume, determination of filling status based on CVP and PAOP is difficult in IAH [9-11]. Therefore, a second aim was to study the effect of elevated IAP on different clinical markers of preload status: pressure-based parameters (CVP, $\mathrm{PAOP})$, transmural pressure-based parameters incorporating a correction for pleural pressure and volumetric parameters. This study was conducted in a porcine model of elevated IAP. 


\section{Methods}

2.1. Animal Instrumentation. This study was performed in accordance with the guidelines and after explicit approval of the local Institutional Ethics Committee on Animal Care and Use. After overnight fasting with free access to water, 11 domestic pigs (mean body weight of $45 \pm 5 \mathrm{~kg}$ ) were anaesthetized with $4 \mathrm{mg} / \mathrm{kg}$ of tiletamine and $4 \mathrm{mg} / \mathrm{kg}$ zolazepam (Zoletil, Virbac, Barneveld, The Netherlands), in combination with $3 \mathrm{mg} / \mathrm{kg}$ xylazine (Xyl-M, VMD, Breendonk, Belgium), all intramuscularly. A polyethylene catheter with a thin-walled, flaccid latex balloon $(10 \mathrm{~cm}$ long) sealed over one end of the catheter (International Medical, Zutphen, The Netherlands) was passed through the snout into the oesophagus, the pigs being in supine position without restraints. The catheter was first positioned into the stomach. This position was confirmed by an increase in the balloon pressure during a spontaneous inspiratory effort. The catheter was then withdrawn to the point where pressure swings reversed direction resulting in negative inspiratory pressures, indicating that the balloon had entered the thoracic cavity. Finally, the catheter was withdrawn another $10 \mathrm{~cm}$ and was fixed to the snout. Pigs were intubated and mechanically ventilated (Servo 900C, Siemens) using an inspiratory oxygen concentration ( $\mathrm{fiO} 2)$ of 0.5 , a constant tidal volume (TV) of $9 \mathrm{~mL} / \mathrm{kg}$, an inspiration/expiration ratio of $1: 2$ and a positive end-expiratory pressure (PEEP) of $5 \mathrm{cmH} 2 \mathrm{O}$. The respiratory rate was adjusted to maintain arterial $\mathrm{pCO}_{2}$ between $35-45 \mathrm{mmHg}$. Anaesthesia was maintained by continuous infusion of $7 \mathrm{mg} \cdot \mathrm{kg}^{-1} \cdot \mathrm{h}^{-1}$ of propofol (Diprivan, AstraZeneca, Brussels, Belgium), $0.1 \mathrm{mg} \cdot \mathrm{kg}^{-1} \cdot \mathrm{h}^{-1}$ pancuronium (Pavulon, Organon, The Netherlands) and with a bolus of $0.25 \mathrm{mg} \cdot \mathrm{kg}^{-1}$ buprenorphine (Temgesic, ScheringPlough, Brussels, Belgium) every 3 h. A 5 F thermistor-tipped catheter (Pulsion Medical Systems, Munich, Germany) was placed in the descending aorta via the left femoral artery. Via a midneck incision, a pulmonary artery thermodilution catheter (Baxter Healthcare Corp., Irvine, CA, USA) was placed in the pulmonary artery via the right external jugular vein. Hartmann/Ringer lactate at $4 \mathrm{~mL} \cdot \mathrm{kg}^{-1} \cdot \mathrm{h}^{-1}$ (Viaflex, Baxter, Lessines, Belgium), glucose $50 \%$ at $0.1 \mathrm{~g} \cdot \mathrm{kg}^{-1} \cdot \mathrm{h}^{-1}$, and HAES-steril at $5 \mathrm{~mL} \cdot \mathrm{kg}^{-1} \cdot \mathrm{h}^{-1}$ (FreeFlex, Fresenius, Friedberg, Germany) were administered as continuous infusion together with anaesthetic drugs via an additional 3lumen catheter in the left external jugular vein. Core temperature was maintained at $38.5 \pm 0.5^{\circ} \mathrm{C}$ using a heating blanket. A midline laparotomy was performed. An aircapsule pressure catheter (Spiegelberg, Hamburg, Germany) was placed in the right lower abdomen, and a drain was placed in the left lower abdomen for intraperitoneal saline infusion. A catheter was inserted into the bladder by a transdermal suprapubic puncture for urine collection. All catheters were exteriorised and the laparotomy was carefully closed in two layers.

2.2. Measurements and Calculations: Pulmonary Mechanics and Pulmonary Function. Peak inspiratory pressure (PIP) and end-inspiratory plateau pressure (Pplat) were recorded from the ventilator. Esophageal pressure (Peso), as a surrogate for pleural pressure, was determined endinspiratory (eiPeso) and end-expiratory (eePeso) by inflating the balloon with $0.75 \mathrm{~mL}$ of air. Total compliance of the respiratory system (Crs) was calculated as TV/(Pplat-PEEP). Chest wall compliance $(\mathrm{Ccw})$ was calculated as $\mathrm{TV} * 0.75 /$ (eiPeso-eePeso) and lung compliance (Clung) as TV/ ((Pplat-PEEP)-((eiPeso-eePeso)/0.75)). Because airway pressures are expressed in $\mathrm{cmH} 2 \mathrm{O}$ and esophageal pressures in $\mathrm{mmHg}$, we used 0.75 to convert units when necessary. Blood samples were taken from the left femoral artery and arterial blood gas values ( $\mathrm{paO} 2$, paCO2) were determined in a blood gas analyser (ABL System 625, Radiometrics Medical, Copenhagen, Denmark).

\subsection{Measurements and Calculations: Global Hemodynamics.} Mean arterial pressure (MAP), heart rate (HR), central venous pressure (CVP), mean pulmonary artery pressure (MPAP), and end-expiratory pulmonary artery occlusion pressure (PAOP) were measured. All pressures were zeroed at the midchest level. No vasoactive drugs were administered. To assess filling pressures in the context of elevated IAP, we calculated transmural mean CVP (tCVP) as CVP-mPeso, with mPeso being calculated as $((2 *$ eePeso + eiPeso $) / 3)$. Transmural end-expiratory pulmonary artery occlusion pressure (tPAOP) as POAP-eePeso. After injecting a bolus of $15 \mathrm{~mL}$ of iced saline into the superior vena cava, stroke index (SI) was calculated from cardiac index (CI) as $\mathrm{CI} / \mathrm{HR}$. CI was determined from transpulmonary thermal indicator dilution at the femoral artery. Systemic vascular resistance (SVRI) was calculated as (MAP-CVP)/CI. Based on the mean transit time and the exponential downslope time of the thermodilution curve, global end-diastolic volume index (GEDVI) and extravascular lung water index (EVLWI) were calculated with the PICCO system [12-14]. SI and intrathoracic blood volume are reported as the average of three repeated measurements lying within $10 \%$ range. Indices are calculated based on bodyweight. Intra-abdominal pressure (IAP) was measured with an IAP monitor (Spiegelberg, Hamburg, Germany) [15].

2.4. Experimental Protocol. After a stabilization period of 2 hours following surgical preparation, a baseline measurement was made and respiratory settings and infusions rates were kept constant during the rest of the protocol. Then IAP was increased sequentially to 10,20 , and $30 \mathrm{mmHg}$ by infusing warmed $\left(38^{\circ} \mathrm{C}\right)$ saline into the peritoneal cavity. The animals were maintained at each IAP level for 45 minutes and then measurements were obtained. After the measurement at $30 \mathrm{mmHg}$, saline was drained. 45 minutes later, the last dataset was acquired and animals were sacrificed by hypertonic potassium chloride injection under deep anaesthesia.

2.5. Statistical Analysis. Results are derived from one measurement per IAP level per pig and are expressed as mean \pm SEM. Since data were not normally distributed, a Friedman analysis of variance was used to analyze the evolution of parameters over different IAP levels. In case of significant results, a post hoc multiple comparison analysis was 


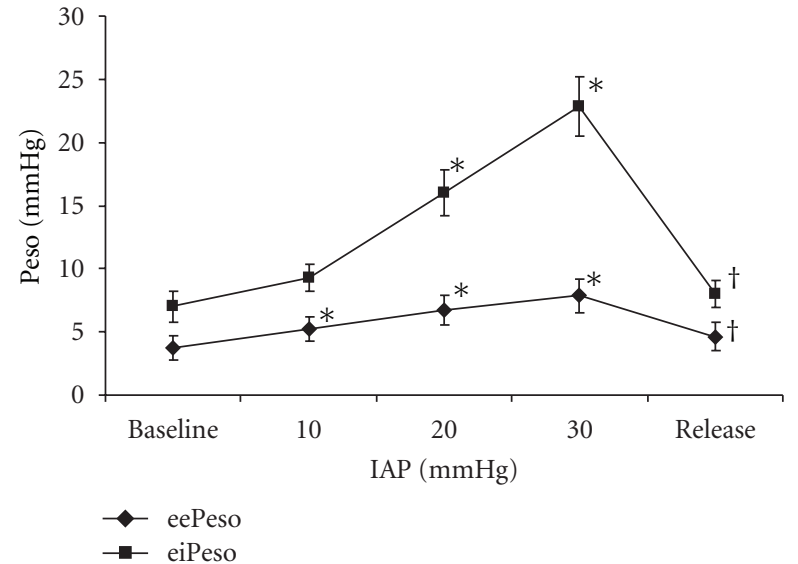

FIGURE 1: Effect of increasing intra-abdominal pressure (IAP) on end-expiratory esophageal pressure (eePeso) and end-inspiratory esophageal pressure (eiPeso). Baseline $=6 \mathrm{mmHg}$, release $=$ $5 \mathrm{mmHg}$. Data are expressed as mean \pm SEM. ${ }^{*} P=0.04$ versus baseline, ${ }^{\dagger} P=0.04$ versus IAP $=30$.

performed using a Bonferroni correction. For correlations, Spearman coefficient of correlation $(r)$ was calculated (Statistica 7.0, StatSoft Inc., Tulsa, USA). For all tests, $P \leq 0.05$ was considered statistically significant.

\section{Results}

3.1. Effects of Elevated IAP on Abdominothoracic Pressure Transmission, Pulmonary Compliance, and Pulmonary Function. IAP was $6 \pm 1 \mathrm{mmHg}$ before (baseline) and $5 \pm$ $1 \mathrm{mmHg}$ at the end of the experiment (release). Both, eePeso and eiPeso increased significantly with increasing IAP. The increase in eiPeso was much more pronounced (Figure 1). After abdominal decompression, eePeso and eiPeso returned to baseline values. Using eePeso, $17 \%$ of IAP was transmitted from the abdominal to the thoracic compartment as shown by linear regression with eePeso $=0.17 * \mathrm{IAP}+4.2(r=0.6$, $P=0.00001)$. Using eiPeso or mPeso, transmission was $62 \%$ and $33 \%$, respectively, $(P=0.000001)$. With increasing IAP, both Crs and Ccw decreased significantly (Figure 2). The decrease was more pronounced for $\mathrm{Ccw}$ and showed a strong inverse correlation with IAP $(r=-0.84, P=$ $0.00001)$. Clung did not change significantly over the whole IAP range. After decompression, $\mathrm{Ccw}$ returned to baseline but Crs remained significantly lower than baseline.

Other effects of increasing IAP on pulmonary dynamics and function are summarised in Table 1. As expected, Pplat and PIP increased significantly with increasing IAP, with Pplat being $0.52 * \mathrm{IAP}+8(r=0.87, P=0.000001)$. PaCO2, $\mathrm{PaO} 2 / \mathrm{fiO} 2$, and EVLWI were not significantly affected by increasing IAP.

3.2. Effects of IAP on Global Hemodynamics. While heart rate (HR) remained constant over the whole IAP range, SI decreased with increasing IAP (Table 1). Simultaneously, mean systemic and pulmonary pressures increased significantly in parallel with a significant rise in SVRI. After IAP

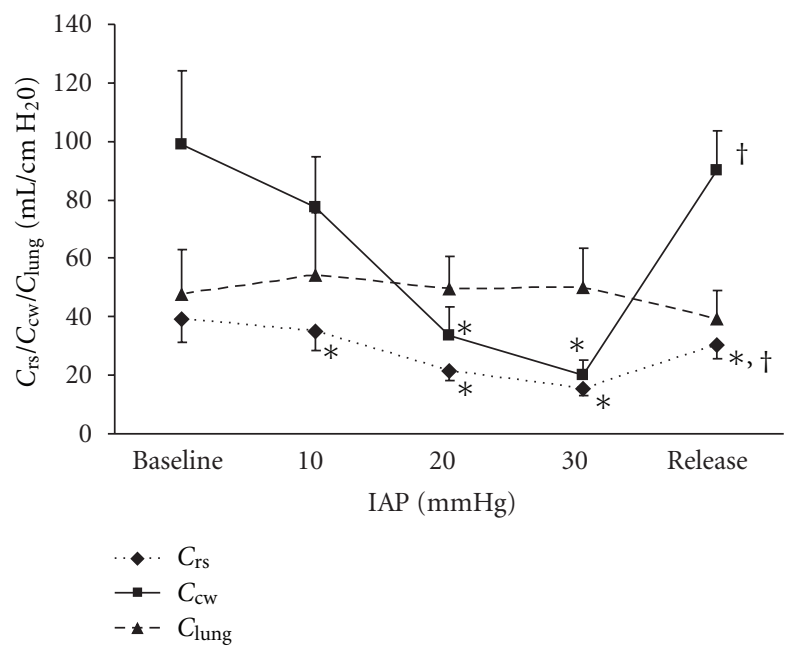

FIGURE 2: Effect of increasing intra-abdominal pressure (IAP) on respiratory system compliance (Crs), chest wall compliance $(\mathrm{Ccw})$, and lung compliance (Clung). Baseline $=6 \mathrm{mmHg}$, release $=$ $5 \mathrm{mmHg}$. Data are expressed as mean \pm SEM. ${ }^{*} P=0.04$ versus baseline, ${ }^{\dagger} P=0.04$ versus IAP $=30$.

release, MAP and SVRI showed a decrease towards baseline level, although MPAP stayed 30\% above baseline value (Table 1). Filling pressures like CVP and PAOP also increased significantly in response to increasing IAP (Figure 3 ), with $\mathrm{CVP}=0.22 * \mathrm{IAP}+5.6(r=0.65, P=0.000001)$ and $\mathrm{PAOP}=0.18 * \mathrm{IAP}+6.9(r=0.49, P=0.001) . \mathrm{CVP}$ and PAOP both returned nearly to baseline values after decompression. Transmural filling pressures (tCVP and tPAOP) showed a decreasing trend with increasing IAP (Figure 3 ). While PAOP and CVP correlated inversely with SI $(r=$ $-0.31, P=0.02)$, tCVP and tPAOP showed a moderate positive correlation with SI $(r=0.36, P=0.03)$. GEDVI, as a volumetric preload estimator, remained quite constant over the different levels of IAP but correlated better with SI $(r=0.52, P=0.04)$ than transmural filling pressures did.

\section{Discussion}

The main findings of this study are, first, that measurement of transfer of IAP to the thoracic compartment is dependent on the stage of the respiratory cycle in which pleural pressures are measured. Second, IAH results in a decreased respiratory system compliance due to a steep decrease of chest wall compliance. Third, transmural filling pressures and GEDVI are clearly superior to classical cardiac filling pressures such as CVP or PAOP in the assessment of cardiac preload at high intra-abdominal pressures.

4.1. Effects of IAP on Abdominothoracic Pressure Transmission, Pulmonary Compliance, and Pulmonary Function. We observed a transmission of IAP to the thoracic compartment of $17 \%, 33 \%$, and $62 \%$, when using end-expiratory, mean, or end-inspiratory pleural pressure, respectively. This is in agreement with others reporting IAP transmissions of $35-60 \%$ using mean pleural pressure and $55-70 \%$ based 
TABLE 1: Effect of increasing intra-abdominal pressure (IAP) on pulmonary mechanics, pulmonary function, and global hemodynamics. Pplat plateau airway pressure, PIP peak inspiratory pressure, EVLWI extravascular lung water index, HR heart rate, MAP mean arterial pressure, MPAP mean pulmonary arterial pressure, SI stroke index, SVRI systemic vascular resistance index, and GEDVI global end-diastolic volume index. Data are expressed as mean \pm SEM.

\begin{tabular}{|c|c|c|c|c|c|}
\hline IAP (mmHg) & Baseline & 10 & 20 & 30 & Release \\
\hline \multicolumn{6}{|l|}{ Pulmonary data } \\
\hline Pplat $(\mathrm{cmH} 2 \mathrm{O})$ & $16 \pm 2$ & $17 \pm 2$ & $24 \pm 2^{*}$ & $32 \pm 2^{*}$ & $19 \pm 2^{*, \dagger}$ \\
\hline PIP (cmH2O) & $19 \pm 1$ & $20 \pm 2$ & $27 \pm 2 *$ & $34 \pm 2 *$ & $21 \pm 2^{\dagger}$ \\
\hline EVLWI (mL/kg) & $13 \pm 2$ & $14 \pm 2$ & $15 \pm 2$ & $14 \pm 3$ & $14 \pm 2$ \\
\hline $\mathrm{paO} 2 / \mathrm{fiO} 2(\mathrm{mmHg})$ & $487 \pm 13$ & $467 \pm 14$ & $487 \pm 12$ & $394 \pm 15$ & $376 \pm 15$ \\
\hline $\mathrm{paCO} 2(\mathrm{mmHg})$ & $39 \pm 2$ & $34 \pm 2$ & $38 \pm 2$ & $38 \pm 1$ & $39 \pm 1$ \\
\hline \multicolumn{6}{|l|}{ Hemodynamic data } \\
\hline $\mathrm{HR}$ (bpm) & $92 \pm 5$ & $97 \pm 4$ & $92 \pm 4$ & $93 \pm 4$ & $92 \pm 4$ \\
\hline MAP (mmHg) & $93 \pm 4$ & $91 \pm 5$ & $98 \pm 5$ & $104 \pm 5^{*}$ & $98 \pm 5$ \\
\hline MPAP (mmHg) & $26 \pm 2$ & $27 \pm 2$ & $31 \pm 3^{*}$ & $37 \pm 5^{*}$ & $34 \pm 2^{*, \dagger}$ \\
\hline $\mathrm{SI}(\mathrm{mL} / \mathrm{kg})$ & $1.2 \pm 0.5$ & $1.3 \pm 0.5^{*}$ & $1.2 \pm 0.5$ & $1.0 \pm 0.5^{\ddagger}$ & $1.2 \pm 0.5^{\dagger}$ \\
\hline SVRI (dynes $\cdot \mathrm{s} / \mathrm{cm}^{5} \cdot \mathrm{kg}$ ) & $35 \pm 4$ & $28 \pm 4^{*}$ & $34 \pm 5$ & $41 \pm 5^{\ddagger}$ & $34 \pm 5^{\dagger}$ \\
\hline GEDVI (mL/kg) & $15.5 \pm 1.9$ & $17.0 \pm 3.1$ & $16.6 \pm 3.0$ & $14.9 \pm 2.7$ & $17 \pm 3.7$ \\
\hline
\end{tabular}

For pulmonary data: ${ }^{*} P=0.025$ versus baseline, ${ }^{\dagger} P=0.015$ versus IAP $=30$.

For hemodynamic data: ${ }^{*} P=0.04$ versus baseline, ${ }^{\dagger} P=0.05$ versus IAP $=30,{ }^{\ddagger} P=0.05$ versus IAP $=10$. All data without symbols indicate a $P$ value of $>0.05$.

on end-inspiratory pleural pressure assessment $[9,16-18]$. Quintel et al. described no effect on end-expiratory pleural pressure in pigs, but IAP was increased only to $15 \mathrm{mmHg}$ [19]. Although some controversy exists on the accuracy of Peso measurement, several authors found Peso to be a good estimator of pleural pressure in the middle lung [20-23]. Moreover, the fact that we found similar fractions of IAP transmission to intrathoracic vascular pressures $(22 \%$ and $18 \%$ of IAP was transmitted to CVP and end-expiratory PAOP, resp.) and ventilator pressures (52\% of IAP was transmitted to Pplat) as to Peso independently confirms our findings on Peso data.

We observed reduced Crs with increasing IAP. This reduction in Crs was largely attributable to a decrease in Ccw. These findings are in agreement with others, showing similar decreases in $\mathrm{Ccw}$ during $\mathrm{IAH}$, due to elevation of the diaphragm, increasing diaphragmatical stiffness and pleural effusion $[6,18,19,24,25]$. Although EVLWI did not change, suggesting the absence of important alveolar edema, Clung and $\mathrm{paO} 2$ tend to decrease at high IAP and after decompression, indeed suggesting initial degradation of pulmonary function. Data from others clearly show that IAH results in pulmonary dysfunction due to reduction of lung volumes, formation of atelectasis and promotion of lung edema [26]. In this study, the duration of applied IAH is probably too short to observe these effects on pulmonary function and this is one of the main limitations of this study.

4.2. Effects of IAP on Global Hemodynamics. Heart rate (HR) remained constant throughout the experiment, probably due to effects of anesthesia. SI showed an initial increase (8\%) at $10 \mathrm{mmHg}$ of IAP, followed by a decrease (22\%) at $30 \mathrm{mmHg}$ of IAP. In agreement with others, we hypothesize that this evolution in SI resulted from an initial autotransfusion effect from the abdominal compartment, followed by a decrease in venous return in combination with an increased afterload at high IAP [27]. Filling status clearly influences the relationship between IAP and SI. Kashtan et al. reported a decrease in $\mathrm{CO}$ of $53 \%$ in hypovolemic and $17 \%$ in normovolemic dogs when increasing IAP to $40 \mathrm{mmHg}$ by abdominal fluid infusion [8]. Moreover, these authors showed that intravascular volume expansion (with approximately one third of the original intravascular volume) restored $\mathrm{CO}$ to normal during IAH $[9,28]$.

The importance of accurate preload assessment in managing cardiovascular dysfunction in IAH raises concern about the reliability of pressure-based preload assessment in patients with IAH. Elevated intrathoracic pressures, as a result of transdiaphragmatic IAP transmission, have been demonstrated to erroneously increase PAOP and CVP, with low CO $[9,10]$. Our data have confirmed this by showing a strong correlation between Peso and CVP $(r=0.75)$ or PAOP $(r=0.7)$. We even observed weak but significant negative correlations between CVP or PAOP and SI, making $\mathrm{CVP}$ and PAOP not useful as preload estimators. Our findings are in good agreement with others demonstrating a moderate negative correlation between CVP and $\mathrm{CO}$ in a porcine model of long-term IAH $(30 \mathrm{mmHg})$ [29-31].

Some authors advocated the use of transmural filling pressures to assess preload in the setting of IAH or high PEEP $[9,20,31]$. By using Peso to estimate pericardial pressure, we found both tCVP and tPAOP to decrease when IAP was increased. In addition, we could demonstrate tCVP and tPAOP to be weakly $(r=0.37)$ correlated with SI. Other investigators studied volumetric parameters to assess preload in the setting of high interfering pressures [30-32]. Leucke et al. showed intrathoracic blood volume (ITBV) and right ventricular end-diastolic volume (RVEDV) to be good estimates of cardiac preload at high intrathoracic pressures by comparing them with left ventricular end-diastolic volume (LVEDV), the "true cardiac preload," as measured by rapid acquisition computed tomography [32]. In agreement with 


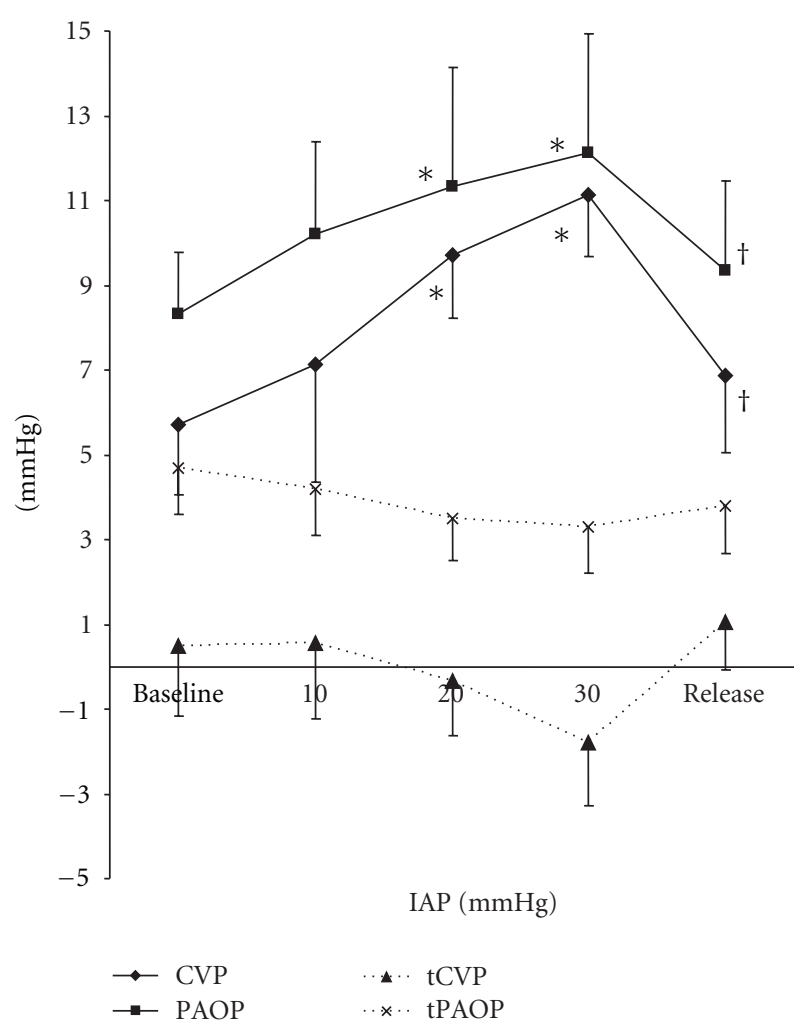

FIGURE 3: Effect of increasing intra-abdominal pressure (IAP) on central venous pressure (CVP), end-expiratory pulmonary artery occlusion pressure (PAOP) and transmural filling pressures (tCVP and $\mathrm{tPAOP}$ ). Baseline $=6 \mathrm{mmHg}$, release $=5 \mathrm{mmHg}$. Data are expressed as mean \pm SEM. ${ }^{*} P=0.04$ versus baseline, ${ }^{\dagger} P=0.05$ versus IAP $=30$.

these observations, we found GEDVI to be the best preload estimator during IAH, as it correlated best with SI $(r=$ 0.52). In our porcine model of IAH, GEDVI first raised from $15.5 \pm 1.8$ to $17.0 \pm 3.1 \mathrm{~mL} / \mathrm{kg}$ at IAP $=10 \mathrm{mmHg}$ and then decreased towards $14.9 \pm 2.7 \mathrm{~mL} / \mathrm{kg}$ at IAP of $30 \mathrm{mmHg}(P=0.07)$. Our findings are in agreement with data in healthy pigs reported by Quintel et al., showing a significant increase in ITBVI from $18.1 \pm 1.6$ to $22.4 \pm$ $2.9 \mathrm{~mL} / \mathrm{kg}$, when increasing IAP to $13 \mathrm{cmH} 2 \mathrm{O}$. A further IAP increase to $26 \mathrm{cmH} 2 \mathrm{O}$ reduced ITBVI to $17.9 \pm 2.2 \mathrm{~mL} / \mathrm{kg}$ $(P=0.023)[19]$. The fact that changes in GEDVI did not reach significance may not surprise since our study was not designed to change intravascular filling status and since SI is determined not only by preload, but also by afterload and cardiac contractility. Importantly, in this study, we did not assess the clinical effect of changing hemodynamics on organ perfusion by measuring lactate or base deficit levels, being another limitation of this study.

\section{Conclusion}

In a porcine model of acute IAH, we found transmission of IAP to the thoracic compartment to be clearly dependent on the stage of the respiratory cycle. Second, IAH results in a decreased respiratory system compliance due to a steep decrease of chest wall compliance. Moreover, GEDVI as well as transmural filling pressures are clearly superior to classical cardiac filling pressures in the assessment of cardiac preload at high intra-abdominal pressures.

\section{Acknowledgments}

The authors thank Dr. Stephen Fieuws for his advice in the statistical analysis and Dr. Arne Neyrinck and Dr. Caroline Van de Wauwer for their surgical assistance. P. Claus is a postdoctoral fellow of the Fund of Scientific Research Flanders (FWO-Vlaanderen, Belgium).

\section{References}

[1] M. L. N. G. Malbrain, D. Chiumello, P. Pelosi et al., "Incidence and prognosis of intraabdominal hypertension in a mixed population of critically ill patients: a multiple-center epidemiological study," Critical Care Medicine, vol. 33, no. 2, pp. 315-322, 2005.

[2] M. L. Malbrain, M. L. Cheatham, A. Kirkpatrick et al., "Results from the International Conference of Experts on Intra-abdominal Hypertension and Abdominal Compartment Syndrome. I. Definitions," intensive care medicine, vol. 32, no. 11 , pp. 1722-1732, 2006.

[3] M. L. N. G. Malbrain, D. Deeren, and T. J. R. De Potter, "Intraabdominal hypertension in the critically ill: it is time to pay attention," Current Opinion in Critical Care, vol. 11, no. 2, pp. 156-171, 2005.

[4] D. J. Cullen, J. P. Coyle, R. Teplick, and M. C. Long, "Cardiovascular, pulmonary, and renal effects of massively increased intra-abdominal pressure in critically ill patients," Critical Care Medicine, vol. 17, no. 2, pp. 118-121, 1989.

[5] M. Sugrue, "Abdominal compartment syndrome," Current Opinion in Critical Care, vol. 11, no. 4, pp. 333-338, 2005.

[6] T. Mutoh, W. J. E. Lamm, L. J. Embree, J. Hildebrandt, and R. K. Albert, "Abdominal distension alters regional pleural pressures and chest wall mechanics in pigs in vivo," Journal of Applied Physiology, vol. 70, no. 6, pp. 2611-2618, 1991.

[7] J. J. Rouby, L. Puybasset, A. Nieszkowska, and Q. Lu, "Acute respiratory distress syndrome: lessons from computed tomography of the whole lung," Critical Care Medicine, vol. 31, no. 4, pp. S285-S295, 2003.

[8] J. Kashtan, J. F. Green, E. Q. Parsons, and J. W. Holcroft, "Hemodynamic effects of increased abdominal pressure," Journal of Surgical Research, vol. 30, no. 3, pp. 249-255, 1981.

[9] P. C. Ridings, G. L. Bloomfield, C. R. Blocher, and H. J. Sugerman, "Cardiopulmonary effects of raised intra-abdominal pressure before and after intravascular volume expansion," Journal of Trauma, vol. 39, no. 6, pp. 1071-1075, 1995.

[10] M. L. Cheatham, L. D. Nelson, M. C. Chang, and K. Safcsak, "Right ventricular end-diastolic volume index as a predictor of preload status in patients on positive end-expiratory pressure," Critical Care Medicine, vol. 26, no. 11, pp. 1801-1806, 1998.

[11] M. L. Malbrain and M. L. Cheatham, "Cardiovascular effects and optimal preload markers in intra-abdominal hypertension," in Yearbook of Intensive Care and Emergency Medicine, J.L. Vincent, Ed., pp. 519-543, Springer, Berlin, Germany, 2004.

[12] J. Renner, M. Gruenewald, P. Brand et al., "Global enddiastolic volume as a variable of fluid responsiveness during 
acute changing loading conditions," Journal of Cardiothoracic and Vascular Anesthesia, vol. 21, no. 5, pp. 650-654, 2007.

[13] J. Renner, P. Meybohm, M. Gruenewald et al., "Global enddiastolic volume during different loading conditions in a pediatric animal model," Anesthesia and Analgesia, vol. 105, no. 5, pp. 1243-1249, 2007.

[14] F. Michard, "Bedside assessment of extravascular lung water by dilution methods: temptations and pitfalls," Critical Care Medicine, vol. 35, no. 4, pp. 1186-1192, 2007.

[15] T. J. R. De Potter, H. Dits, and M. L. N. G. Malbrain, "Intraand interobserver variability during in vitro validation of two novel methods for intra-abdominal pressure monitoring," Intensive Care Medicine, vol. 31, no. 5, pp. 747-751, 2005.

[16] U. B. Windberger, R. Auer, F. Keplinger et al., "The role of intra-abdominal pressure on splanchnic and pulmonary hemodynamic and metabolic changes during carbon dioxide pneumoperitoneum," Gastrointestinal Endoscopy, vol. 49, no. 1, pp. 84-91, 1999.

[17] G. L. Bloomfield, P. C. Ridings, C. R. Blocher, A. Marmarou, and H. J. Sugerman, "A proposed relationship between increased intra-abdominal, intrathoracic, and intracranial pressure," Critical Care Medicine, vol. 25, no. 3, pp. 496-503, 1997.

[18] G. Citerio, E. Vascotto, F. Villa, S. Celotti, and A. Pesenti, "Induced abdominal compartment syndrome increases intracranial pressure in neurotrauma patients: a prospective study," Critical Care Medicine, vol. 29, no. 7, pp. 1466-1471, 2001.

[19] M. Quintel, P. Pelosi, P. Caironi et al., "An increase of abdominal pressure increases pulmonary edema in oleic acidinduced lung injury," American Journal of Respiratory and Critical Care Medicine, vol. 169, no. 4, pp. 534-541, 2004.

[20] R. H. Kallet, J. A. Katz, J. F. Pittet et al., "Measuring intraesophageal pressure to assess transmural pulmonary arterial occlusion pressure in patients with acute lung injury: a case series and review," Respiratory Care, vol. 45, no. 9, pp. 10721084, 2000.

[21] J. J. Marini, R. O'Quin, B. H. Culver, and J. Butler, "Estimation of transmural cardiac pressures during ventilation with PEEP," Journal of Applied Physiology, vol. 53, no. 2, pp. 384-391, 1982.

[22] G. Dechman, J. Sato, and J. H. T. Bates, "Factors affecting the accuracy of esophageal balloon measurement of pleural pressure in dogs," Journal of Applied Physiology, vol. 72, no. 1, pp. 383-388, 1992.

[23] P. Pelosi, M. Goldner, A. McKibben et al., "Recruitment and derecruitment during acute respiratory failure: an experimental study," American Journal of Respiratory and Critical Care Medicine, vol. 164, no. 1, pp. 122-130, 2001.

[24] V. M. Ranieri, N. Brienza, S. Santostasi et al., "Impairment of lung and chest wall mechanics in patients with acute respiratory distress syndrome: role of abdominal distension," American Journal of Respiratory and Critical Care Medicine, vol. 156, no. 4, pp. 1082-1091, 1997.

[25] L. Gattinoni, P. Pelosi, P. M. Suter, A. Pedoto, P. Vercesi, and A. Lissoni, "Acute respiratory distress syndrome caused by pulmonary and extrapulmonary disease: different syndromes?" American Journal of Respiratory and Critical Care Medicine, vol. 158, no. 1, pp. 3-11, 1998.

[26] P. Pelosi, M. Quintel, and M. L. N. G. Malbrain, "Effect of intra-abdominal pressure on respiratory mechanics," Acta Clinica Belgica, vol. 62, no. 1, pp. 78-88, 2007.

[27] M. Diamant, J. L. Benumof, and L. J. Saidman, "Hemodynamics of increased intra abdominal pressure: interaction with hypovolemia and halothane anesthesia," Anesthesiology, vol. 48, no. 1, pp. 23-27, 1978.
[28] A. Schachtrupp, G. Lawong, M. Afify, J. Graf, C. Toens, and V. Schumpelick, "Fluid resuscitation preserves cardiac output but cannot prevent organ damage in a porcine model during $24 \mathrm{H}$ of intraabdominal hypertension," Shock, vol. 24, no. 2, pp. 153-158, 2005.

[29] I. Kingma, O. A. Smiseth, M. A. Frais, E. R. Smith, and J. V. Tyberg, "Left ventricular external constraint: relationship between pericardial, pleural and esophageal pressures during positive end-expiratory pressure and volume loading in dogs," Annals of Biomedical Engineering, vol. 15, no. 3-4, pp. 331-346, 1987.

[30] A. Schachtrupp, J. Graf, C. Tons, J. Hoer, V. Fackeldey, and V. Schumpelick, "Intravascular volume depletion in a 24-hour porcine model of intra-abdominal hypertension," Journal of Trauma, vol. 55, no. 4, pp. 734-740, 2003.

[31] M. L. Cheatham, K. Safcsak, E. F. J. Block, and L. D. Nelson, "Preload assessment in patients with an open abdomen," Journal of Trauma, vol. 46, no. 1, pp. 16-22, 1999.

[32] T. Luecke, H. Roth, P. Herrmann et al., "Assessment of cardiac preload and left ventricular function under increasing levels of positive end-expiratory pressure," Intensive Care Medicine, vol. 30, no. 1, pp. 119-126, 2004. 


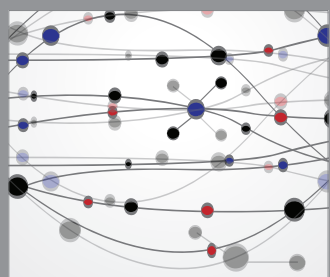

The Scientific World Journal
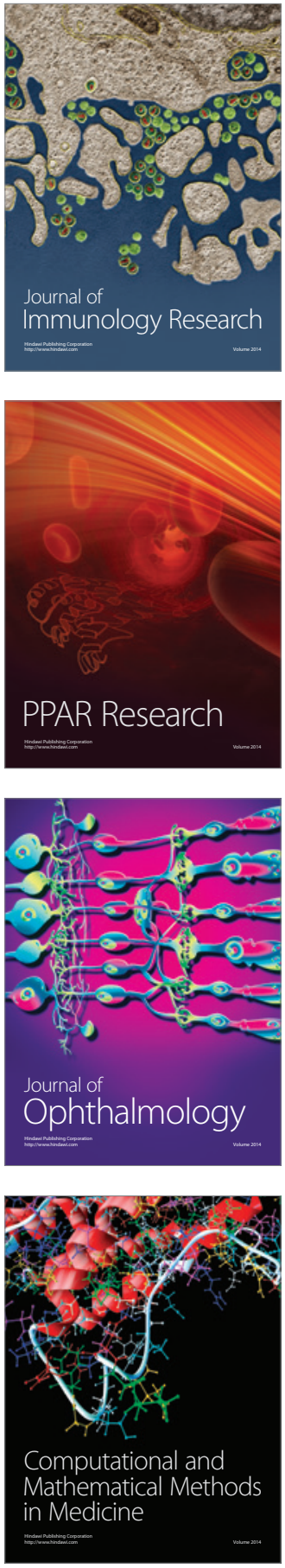

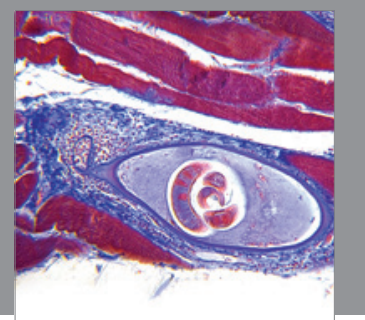

Gastroenterology

Research and Practice
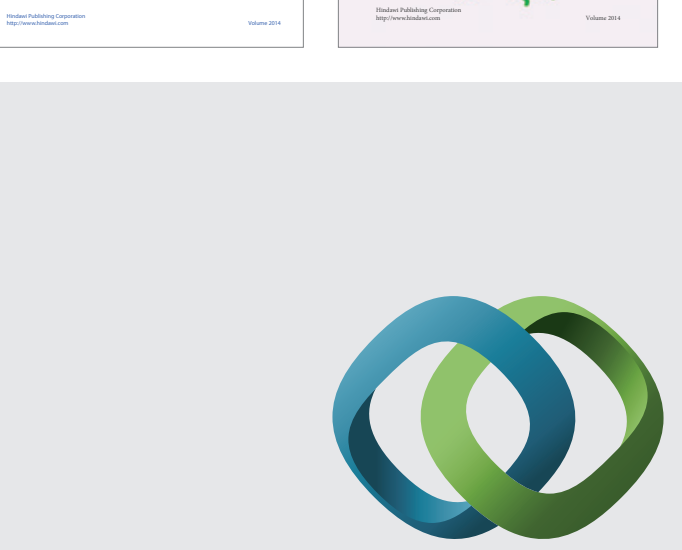

\section{Hindawi}

Submit your manuscripts at

http://www.hindawi.com
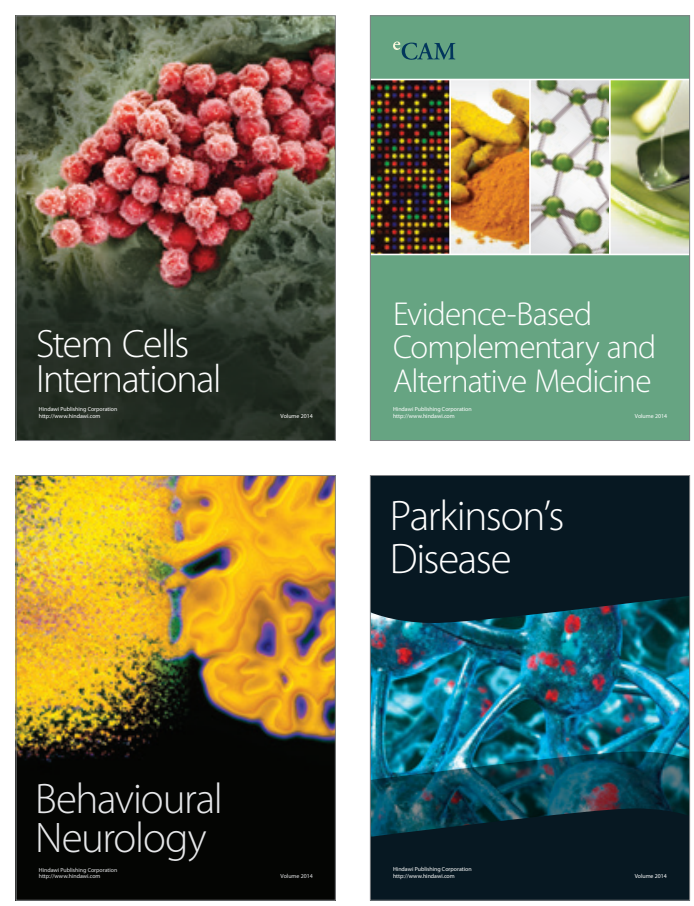

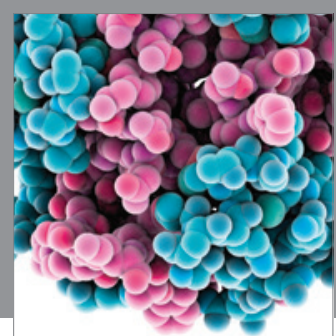

Journal of
Diabetes Research

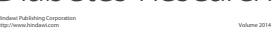

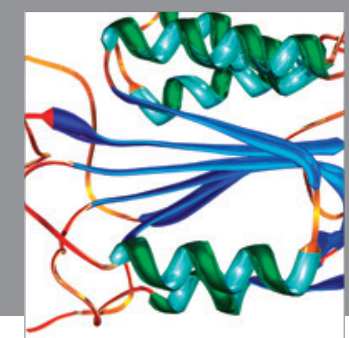

Disease Markers
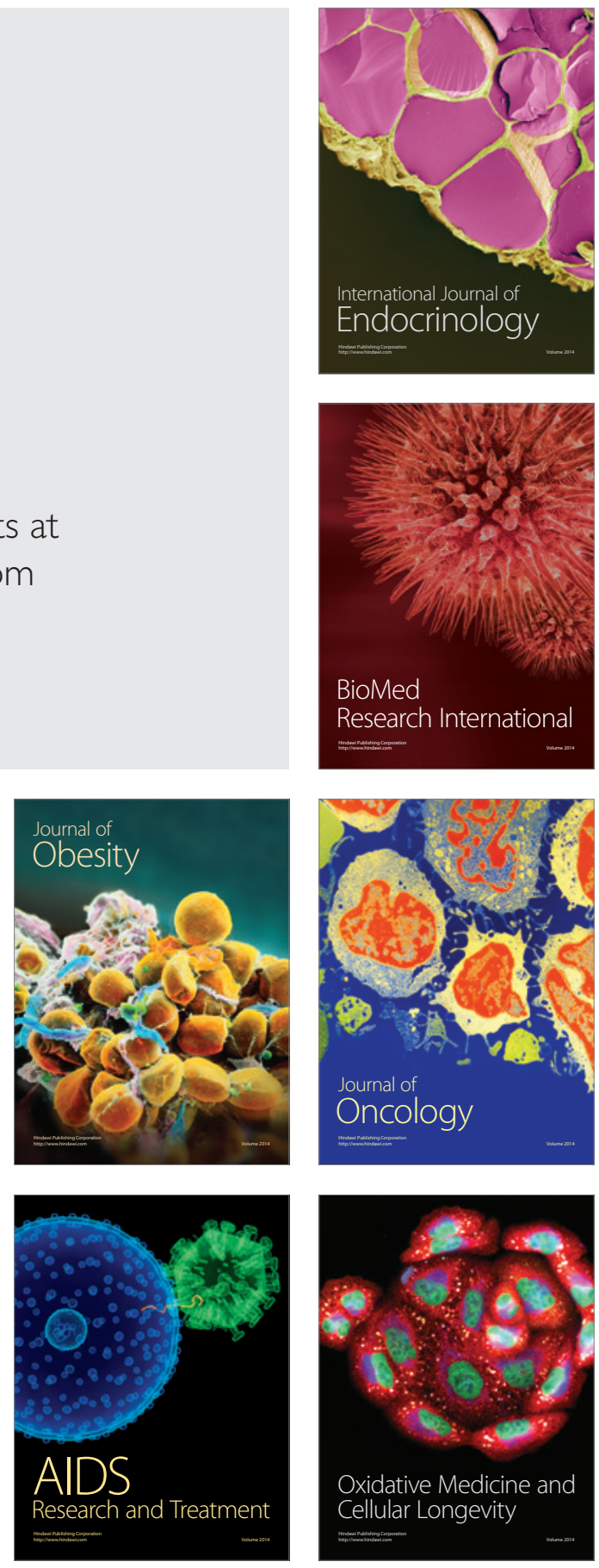